\title{
Précarisation de la profession enseignante au primaire en Côte d'Ivoire
}

\begin{abstract}
Alain Toh
Institut d'ethnosociologie

Université Félix Houphouët-Boigny de Cocody,

Côte d'Ivoire
\end{abstract}

Making primary teaching

precarious in Ivory Coast

doi:10318162/fp.2017.335
$R$ ésumé

La Côte d'Ivoire subit les conséquences des politiques transnationales depuis près d'une vingtaine d'années. L'éducation figure parmi les secteurs les plus affectés. En effet, les données factuelles indiquent que les effets des réformes internationales semblent se traduire en contraintes qui compromettent sérieusement la qualité de l'éducation dans ce pays. Le présent article s'intéresse plus particulièrement aux déclinaisons socioéconomiques des politiques transnationales sur la profession enseignante et notamment sur les conditions de vie et de travail des enseignants de l'enseignement primaire public en Côte d'Ivoire. II prend appui sur une étude visant à documenter et à analyser la précarisation de la profession enseignante dans ce pays, ainsi que les réponses développées par différents acteurs (gouvernement, syndicats, ONG, institutions internationales) pour rétablir la situation.

Mots-clés

Réformes internationales, profession enseignante, informalisation, précarisation, politiques d'ajustement

structurel

Abstract

Côte d'Ivoire has been dealing with the consequences of transnational policies for the past 20 years. Education is one of the hardest hit sectors. Data available suggest that the effects of international reforms have led to constraints that have seriously harmed the quality of education in this country. This article focuses on the socioeconomic effects of transnational policies on the teaching profession, particularly on the living and working conditions of public primary school teachers in Côte d'Ivoire. It draws on a study aimed at documenting and analyzing the process of making primary teaching a precarious profession in this country, as well as the responses developed by various stakeholders (Government, teacher organizations, NGOs, international institutions) to improve the situation.

Keywords

International reforms, teaching profession, informalization, precariousness, structural adjustment policies

\section{Problématique}

Dans les premières années de l'accession de la Côte d'Ivoire à l'indépendance (1960), les pouvoirs publics ont affirmé leur volonté de placer la valorisation des ressources humaines au premier plan de leurs préoccupations (Kouadio, 2001). Soutenues à l'époque par les immenses potentialités économiques, l'éducation et la formation ont très tôt bénéficié d'un important investissement. L'État ivoirien va consacrer à ce secteur stratégique, de 1960 à 1980, plus de $44 \%$ du budget national (Sahi, 2005). La croissance économique a pendant longtemps impulsé pleinement la politique de l'éducation et la formation jusqu'à l'orée des années 1980, avant de connaître un déclin. En effet, la conjoncture de la crise économique mondiale qui a marqué ces années n'a pas épargné la Côte d'Ivoire fortement contrariée par la détérioration des termes d'échange des principaux produits d'exportation, notamment le café et le cacao (Toh et Gauthier, 2011).

Face au marasme économique qui sévissait, les Institutions de Bretton Woods (Banque mondiale et Fonds monétaire international [FMI]) ont imposé des restrictions budgétaires, monétaires et fiscales à un certain nombre de pays d'Afrique subsaharienne, y compris la Côte d'Ivoire (Dowo, 2004). Ce pays a connu une longue traversée marquée par des crises sociopolitiques, des années 1990 jusqu'en 2010-2011, crises qui ont connu leur paroxysme entre 1999 et 2002. Ces crises ont eu des impacts sérieux sur l'éducation et la formation, principalement sur la profession enseignante. Pendant cette période, aucun plan de sauvetage dans le domaine de l'éducation n'a pu véritablement résoudre entièrement la question du malaise de l'école ivoirienne, principalement à cause du contexte délétère qui prévalait 
(Toh et Gauthier, 2011). Les grèves à répétition et les revendications aussi bien des étudiants que des enseignants n'ont pas favorisé un climat scolaire propice à une formation de qualité, mais ont plutôt favorisé des pratiques rendant compte d'une «informalisation » et une précarisation accentuée de la profession enseignante.

Au moment où se déroulent les discussions sur les objectifs post-2015, il est pertinent de s'interroger sur l'impact des politiques transnationales sur la profession enseignante dans les pays en développement. Et le cas de la Côte d'Ivoire semble très instructif à cet égard. Comment est-on arrivé là? Quelles sont les manifestations de cette précarisation de la profession enseignante en Côte d'Ivoire? Quelles réponses sont développées par les différents acteurs (gouvernement, syndicats, ONG, institutions internationales) pour rétablir la situation? Telles sont les questions qui ont guidé l'étude rapportée dans le présent article.

Cet article s'intéresse plus particulièrement à analyser les déclinaisons socioéconomiques des politiques transnationales sur la profession enseignante et notamment sur les conditions de vie et de travail des enseignants de l'enseignement primaire public en Côte d'Ivoire. Il s'agit clairement d'une analyse des implications socioéconomiques de ces politiques sur les conditions de vie et de travail des enseignants. Notre hypothèse est que les réformes internationales ont fortement entamé le développement du capital humain et engendré une informalisation, voire une dégradation accentuée de la profession enseignante en Afrique subsaharienne, et particulièrement en Côte d'Ivoire.

Des études précédentes (Dowo, 2004; Duruflé, 1988; Kouamé, 2014; Kpoumié Monjap, 2005) ont clairement démontré que les programmes d'ajustement structurel ont eu des effets négatifs sur plusieurs composantes du secteur de l'éducation, dont la profession enseignante en Afrique subsaharienne. Toutefois, il existe très peu d'informations, en termes de recherche systématique, à propos des impacts des politiques transnationales sur les conditions de vie et de travail des enseignants. Notre recherche vient donc combler un vide sur la question. À partir des données de terrain, cet article, dans une analyse sociologique fondée sur les logiques d'acteurs, examine l'impact des réformes internationales sur la profession enseignante dans l'enseignement primaire public en Côte d'Ivoire. Cette analyse met l'accent sur l'acteur «social-historique » au sens de la sociologie de Bourdieu. Elle rend compte des logiques d'action en recherchant précisément ce qui fonde les choix des acteurs cibles. Cela vise à comprendre les rationalités qui sont à l'œuvre derrière les opinions des acteurs sociaux relatives aux implications particulières de la mondialisation économique dans les composantes du secteur de l'éducation en Côte d'Ivoire en considérant toutefois que la logique d'action n'est pas une structure causale immuable.

\section{Cadre théorique}

Les principaux concepts qui ont guidé le recueil et structuré l'analyse des données sont les conditions socioéconomiques des enseignants (qui renvoient aux conditions de vie et de travail des enseignants, avec des indicateurs tels que le revenu, le cadre de travail, les rapports avec les élèves, la perception de la profession enseignante, la couverture des besoins en infrastructures, des ratios enseignants / besoins en enseignants, etc.), les dimensions socioculturelles des réformes (renvoient à la perception des enseignants relative aux impacts des politiques internationales dans le secteur primaire, et ce, 
pour saisir le processus de la professionnalisation ou de la déprofessionnalisation de la profession enseignante, etc.). Selon Corcuff (2007), ces concepts rendent compte d'une socio-anthropologie d'inspiration théorique marxienne, amplifiée dans les sciences sociales d'aujourd'hui, qui met l'accent sur « la part idéelle du réel » (Godelier, 1984). Cette approche théorique des concepts a permis de saisir la construction des identités sociales et professionnelles telle que développée dès le début du $\mathrm{XX}^{\mathrm{e}}$ siècle par Alexander M. Carr-Saunders et Paul A. Wilson (1933), prolongée par la suite par Talcott Parsons (1968) et reprise en France par Claude Dubar (2002). C'est à juste titre que, dans une perspective socio-anthropologique orientée sur le constructivisme, les données recueillies ont fait l'objet d'une analyse de contenu. Cela a permis d'avoir une perception différenciée des réalités et des manifestations des réformes internationales sur la profession enseignante dans le secteur primaire public, et de saisir ses impacts réels sur la profession enseignante en Côte d'Ivoire.

\section{Méthodologie}

L'étude sociologique de l'impact des réformes internationales sur la profession enseignante requiert la collecte de données de type quantitatif, mais aussi de nature qualitative. La recherche documentaire a constitué la première étape de collecte des données. Elle a visé, entre autres, à faire une revue des travaux sur la situation de référence du secteur de l'éducation : l'histoire et les généralités des programmes d'ajustement structurels en Côte d'Ivoire, leur contenu et leurs formes, l'application de ces politiques dans le secteur de l'éducation, les initiatives et les discours des organisations politiques et syndicales relatifs à ces réformes, mais également l'évolution de la situation caractérisée par l'après-réformes. Nous avons recensé des articles de presse, des articles et ouvrages scientifiques, des archives d'organisations politiques ou syndicales, des documents administratifs, des rapports d'études, des discours d'acteurs politiques ou institutionnels, et des statistiques que nous avons soumis à une triangulation critique en vue de leur utilisation.

Cette collecte de données primaires a été complétée par des enquêtes sur le terrain dans deux zones choisies en fonction de la carte scolaire et de la situation socioéconomique des différentes régions du pays. Les zones choisies sont Abidjan et Yamoussoukro. Capitale économique de la Côte d'Ivoire, Abidjan absorbe au niveau de la carte scolaire environ la moitié des élèves du pays, tant au niveau du primaire qu'au niveau du secondaire. La moitié des enseignants du primaire exercent dans cette ville qui abrite de nombreuses infrastructures scolaires. Cependant, la répartition semble inégale au niveau des communes. C'est pourquoi nous avons choisi pour les enquêtes deux communes qui présentent des caractéristiques socioéconomiques différentes : Cocody et Abobo. En réalité, Cocody est le quartier résidentiel supposé abriter des infrastructures en nombre suffisant et relativement conforme à l'évolution de la population scolaire. Abobo est un quartier populaire où la demande scolaire est relativement forte, tandis que les infrastructures et le personnel enseignant sont généralement insuffisants. Ces deux situations reflètent la géographie scolaire au niveau de la ville d'Abidjan. Capitale politique de la Côte d'Ivoire, Yamoussoukro reste un grand carrefour où la demande scolaire est forte en raison du conflit armé qui a drainé de nombreux immigrés rendant les infrastructures déjà obsolètes très insuffisantes.

Dans chacune de ces zones, nous avons administré un questionnaire et réalisé des entrevues. Adressé à un échantillon aléatoire de 126 enseignants, le questionnaire visait à saisir les opinions des enseignants du primaire public, leur niveau de connaissance et leurs attentes relativement aux effets des réformes 
transnationales sur l'éducation, et en particulier la profession enseignante, dans ce pays. Les données ainsi recueillies ont fait l'objet d'analyses de statistiques descriptives à l'aide du logiciel SPSS 13.0.

Le second volet de l'enquête, de type qualitatif, a quant à lui été articulé autour des entrevues $(\mathrm{N}=25)$ avec des responsables du secteur de l'éducation (ministère de l'Éducation nationale et inspections d'enseignement primaire), des responsables de syndicats, des comités de gestion des écoles (COGES), des parents d'élèves et des responsables de la Banque mondiale à Abidjan. Ces entrevues semidirigées ont été conduites de façon individuelle auprès des acteurs ci-dessus cités afin de recueillir des informations sur leur expérience des réformes initiée par les institutions financières internationales (Banque mondiale et FMI), les éventuels atouts et faiblesses de ces réformes, ainsi que les propositions visant à développer des réponses appropriées en vue de promouvoir une éducation de qualité dans le secteur primaire public en Côte d'Ivoire. Le contexte sociopolitique particulièrement sensible en Côte d'Ivoire, au moment où nous menions cette étude, conjugué au refus des participants de faire enregistrer leurs propos, a justifié et favorisé l'option de la prise de notes.

\section{Résultats, analyse et discussion}

\section{La profession enseignante avant l'avènement des réformes internationales en Côte d'Ivoire : la période de grâce des enseignants}

Dans cette section, nous nous interrogeons d'abord sur les connaissances générales et spécifiques que les enseignants ont de ces réformes. Nous cherchons ensuite à savoir si ces connaissances sont liées ou non à leur ancienneté professionnelle. Enfin, à partir des indicateurs socioéconomiques (revenu et possibilité d'épargne) et socio-éducatifs (infrastructures scolaires, besoins divers en éducation), nous analysons la représentation sociale que les enseignants eux-mêmes se font de la profession enseignante avant l'avènement des réformes internationales.

\section{Les enseignants, leurs connaissances des réformes internationales et le cadre de travail du maître}

Létude à partir des données factuelles des impacts des réformes internationales dans le secteur de l'éducation nécessite l'analyse du niveau de connaissance des réformes et du cadre de travail du maître.

Les données collectées indiquent que les enseignants du primaire public dans leur majorité ont des informations relatives aux réformes initiées par la Banque mondiale et le FMI. En effet, 74,6 \% des enseignants enquêtés, ayant des expériences professionnelles diverses, ont affirmé connaître ces réformes internationales. Cela permet de dire que les enquêtés sont plus ou moins capables de nous fournir des informations (recherchées) relativement justes, parce qu’en général vécues par ceux-ci sous différentes formes et à divers titres (enseignant, étudiant ou élève). Dans tous les cas, nos interlocuteurs sont des acteurs de l'éducation qui ont plus ou moins une idée de l'impact des politiques transnationales dans le système éducatif en général, et particulièrement sur la profession enseignante en Côte d'Ivoire. 
Les données présentées au tableau 1 indiquent que les enseignants en général (82,54\%) ont une connaissance des réformes initiées par les institutions financières internationales en Côte d'Ivoire. Et ceux qui n'en ont pas affirment avoir tout de même une idée globale de la situation socioéconomique du pays, et surtout de la politique éducative, avant la période d'avènement de ces réformes. Selon M. S. Y., jeune enseignant et responsable du comité de gestion de l'école primaire publique Municipalité 1 de Yamoussoukro : "De façon générale, les jeunes que nous sommes n’avons pas vécu les $P A S^{1}$, mais nous en avons une idée vague issue des causeries avec les autres collègues relativement anciens, mais également par l'intermédiaire de nos organisations syndicales et la presse. On sait que la situation économique des enseignants s'est dégradée depuis quelques années et que les PAS sont indexés dans la mesure où le pays était une grosse locomotive de la sous-région sur le plan économique ».

Il ressort de l'analyse que les enseignants ayant plus de dix ans d'expérience professionnelle ont une bonne connaissance des réformes internationales dans le secteur de l'éducation. Cela rend compte de l'importance de la dimension socio-historique du phénomène à l'étude et de son lien avec les opinions des répondants.

- Le niveau de connaissance des réformes dépend-il de l'ancienneté professionnelle?

\section{Tableau 1}

Niveau de connaissance des réformes en fonction de l'ancienneté professionnelle.

\begin{tabular}{|c|c|c|c|c|c|}
\hline \multirow{3}{*}{$\begin{array}{l}\text { Ancienneté pro- } \\
\text { fessionnelle }\end{array}$} & \multicolumn{4}{|c|}{ Connaissance des PAS } & \multirow{3}{*}{$\begin{array}{l}\text { \% des enquêtés } \\
\text { par ancienneté }\end{array}$} \\
\hline & \multicolumn{2}{|c|}{ Non } & \multicolumn{2}{|c|}{ Oui } & \\
\hline & *VA & *VR & VA & VR & \\
\hline$<$ de 5 ans & 4 & $80 \%$ & 1 & $20 \%$ & $3,97 \%$ \\
\hline 5 à 10 ans & 8 & $72,73 \%$ & 03 & $27,27 \%$ & $8,73 \%$ \\
\hline 10 à 15 ans & 4 & $25 \%$ & 12 & $75 \%$ & $12,7 \%$ \\
\hline 15 à 20 ans & 4 & $10,53 \%$ & 34 & $89,47 \%$ & $30,16 \%$ \\
\hline 20 ans et + & 2 & $3,57 \%$ & 54 & $96,43 \%$ & $44,44 \%$ \\
\hline Total & 22 & $17,46 \%$ & 104 & $82,54 \%$ & $100 \%$ \\
\hline
\end{tabular}

* $(V A=$ Valeur absolue $/ V R=$ Valeur relative $)$

S'il est clair que les enseignants du primaire public, dans leur généralité, ont une connaissance des réformes internationales, il semble intéressant de s'interroger sur leur perception ou leur représentation de la profession enseignante avant l'avènement des réformes. Cette étude des représentations de la profession enseignante, en lien avec la politique éducative, s'appuie particulièrement sur le rapport entre les infrastructures et les besoins en matière d'éducation, l'appréciation des salaires, la possibilité d'épargner et les difficultés particulières rencontrées. Au-delà de la politique éducative qu'ils développent, ces indicateurs permettent également de rendre compte des conditions de travail des enseignants, un des indicateurs caractéristiques de la profession enseignante. En réalité, ce questionnement permet de saisir le secteur éducatif dans un contexte socioéconomique particulier, 
c'est-à-dire l'instauration des mesures particulièrement drastiques des Institutions de Bretton Woods en Côte d'Ivoire. Les résultats des enquêtes montrent qu'avant l'avènement des réformes, les infrastructures éducatives n'étaient pas suffisantes pour répondre aux besoins sociaux en matière d'éducation (voir tableau 2). C'est ce que pensent 56,35\% des répondants contre 40,48\% des enseignants enquêtés qui estiment que ces infrastructures répondaient aux attentes des populations, étant donné le nombre relativement limité des apprenants (26 élèves selon le PASEF, 1998). Et $3,17 \%$ des enquêtés nont pas répondu à cette question. La corrélation indique que cette attitude est certainement liée à leur expérience professionnelle de moins de 5 ans, ce qui ne leur a pas permis d'avoir une idée claire se rapportant aux réalités socio-historiques de l'éducation nationale. L'analyse comparative entre les données quantitatives et qualitatives laisse apparaître que, même si les besoins en infrastructures socio-éducatives étaient insuffisants, leur niveau de couverture et leur entretien en termes de rénovation régulière du cadre de travail des maîtres étaient relativement appréciables.

\section{Tableau 2}

Opinions des maîtres sur le niveau de couverture des besoins en infrastructures scolaires.

\begin{tabular}{lcc}
\hline Opinions & Valeur absolue & Fréquence \\
Insuffisant & 71 & 56,35 \\
Suffisant & 51 & 40,48 \\
Ne sait pas & 4 & 3,17 \\
Total & $\mathbf{1 2 6}$ & $\mathbf{1 0 0}$ \\
\hline
\end{tabular}

\section{La profession enseignante et les conditions socioéconomiques des enseignants : une expression du miracle ivoirien des années fastes}

Les perceptions des salaires des maîtres, de leur possibilité d'épargne et de leurs conditions de travail retenues comme des indicateurs socioéconomiques d'étude de la profession enseignante sont analysées dans une perspective diachronique dans le tableau 3. Ces indicateurs rendent compte des conditions de vie et de travail des maîtres avant l'avènement des réformes transnationales dans le secteur de l'éducation.

Tableau 3

Perceptions des enquêtés sur l'état des salaires.

\begin{tabular}{lcl} 
Appréciation & Valeur absolue & Fréquence \\
Très bon & 24 & 19,05 \\
Bon & 53 & 42,06 \\
Juste acceptable & 36 & 28,57 \\
Mauvais & 13 & 10,32 \\
Total & $\mathbf{1 2 6}$ & $\mathbf{1 0 0 , 0}$ \\
\hline
\end{tabular}


Ces résultats montrent que seulement $10,32 \%$ des enseignants enquêtés pensent que les salaires étaient mauvais. Pour ces enquêtés, le salaire de l'enseignant est resté celui du stagiaire nonobstant le changement de statut, ce qui ne leur permettait pas de subvenir véritablement à leurs besoins sociaux. A contrario, 89,68 \% des enseignants enquêtés ont en général une image positive des salaires avant l'avènement des programmes d'ajustement structurel en Côte d'Ivoire. Selon eux, cette perception est à mettre en rapport avec le coût de la vie qui neétait pas élevé. D’ailleurs, les résultats des entretiens menés auprès des différents acteurs du système éducatif ivoirien ont clairement justifié cette perception de la majorité des enseignants par la valorisation des salaires des fonctionnaires ivoiriens en général, et ceux des enseignants en particulier qui s'est poursuivie jusqu'au début des années 1980, et s'est faite avec l'évolution de la situation économique du pays. En somme, les données indiquent que la majorité des enseignants avait une bonne perception de leur salaire avant l'avènement des programmes d'ajustement structurel. Mais avaient-ils la possibilité d'épargner?

\section{Situation de l'épargne avant les réformes internationales}

En économie, l'épargne constitue un indicateur pertinent de mesure de l'indice de pauvreté dans une société ou dans un ménage. En sociologie, l'épargne traduit une situation relative de transformation matérielle d'un individu, d'un ménage ou d'une société (Baha Bi, 2001). Elle n'implique pas forcément une élimination de la pauvreté, mais elle en constitue une véritable source de réduction, susceptible de générer une assurance dans la gestion des charges socioéconomiques en vue du bien-être social. C'est pourquoi la référence à l'épargne dans cette contribution nous paraît pertinente pour comprendre la situation socioéconomique des enseignants avant les réformes internationales. Le tableau 4 traduit les tendances exprimées par les enseignants enquêtés.

\section{Tableau 4}

L'épargne des enseignants.

\begin{tabular}{lcc}
\hline & Valeur absolue & Fréquence \\
Épargne & 81 & 64,4 \\
Pas d'épargne & 45 & 35,6 \\
Total & $\mathbf{1 2 6}$ & $\mathbf{1 0 0 , 0}$ \\
\hline
\end{tabular}

Les données montrent bien que les salaires des enseignants ${ }^{2}$ leur permettaient d'effectuer des épargnes. Pour $64,4 \%$ des enquêtés, les salaires qu'ils recevaient leur offraient la capacité d'épargner et d'être moins vulnérables aux usuriers. Cette situation permettait aux enseignants « de traduire dans les faits et dans le comportement la noblesse de leur métier " (propos d'un conseiller pédagogique à Yamoussoukro, 29 ans d'ancienneté). 


\section{Appréciation de la politique éducative avant les réformes}

\section{Tableau 5}

Appréciation de la politique éducative dans le secteur primaire public.

\begin{tabular}{lcc}
\hline Appréciations & Valeur absolue & Fréquence \\
Bon & 81 & 64,28 \\
Assez bon & 13 & 10,32 \\
Passable & 23 & 18,25 \\
Mauvais & 9 & 7,15 \\
Total & $\mathbf{1 2 6}$ & $\mathbf{1 0 0 , 0}$ \\
\hline
\end{tabular}

Les données d'enquête indiquent en général que la majorité des acteurs de l'enseignement primaire en Côte d'Ivoire a une perception relativement «bonne » de la politique éducative avant l'avènement des réformes. En effet, sur le plan quantitatif, $64,28 \%$ des enquêtés estiment que la politique éducative ivoirienne avant les réformes était "bonne ", "adaptée », du fait des conditions de vie et de travail des enseignants qui étaient relativement appréciables. Sur le plan qualitatif, cette opinion est partagée généralement par les différents acteurs du système éducatif. Selon M. F. T. L., ex-coordinateur national des activités extrascolaires au ministère de l'Éducation nationale :

Avant l'avènement des politiques internationales en Côte d'Ivoire, la politique éducative ivoirienne était appréciable du fait de la qualité de l'enseignement, des infrastructures, mais surtout les conditions de vie des enseignants ivoiriens qui étaient bonnes; les enseignants bénéficiaient de plusieurs avantages sociaux et financiers; ce qui leur permettait de subvenir à leurs besoins importants de tous ordres. L'environnement de travail était propice à de bons résultats. On notait une quasi-absence de mouvements de grèves, une suffisance et une disponibilité de matériels pédagogiques, l'octroi de primes incitatives pour les directeurs d'école primaire et les conseillers pédagogiques, et un encadrement pédagogique régulier et efficace des enseignants.

En définitive, 74,6 \% des enseignants enquêtés ont une opinion relativement bonne de la politique éducative et de la profession enseignante avant l'avènement des réformes internationales. En revanche, d'autres (soit 26,4\% des enquêtés) estiment que la politique éducative a eu un impact mitigé, voire imperceptible sur la condition enseignante. Ils qualifient la politique éducative avant les réformes de «mauvaise ». Ce dernier groupe d'enquêtés se caractérise particulièrement par sa présence dans des zones jugées difficiles du point de vue professionnel. En effet, les zones du nord de la Côte d'Ivoire sont en général très peu fournies en infrastructures scolaires et socioéconomiques de qualité et les conditions de vie et travail des enseignants du primaire en particulier y sont plus ou moins difficiles : insuffisance voire absence de logements d'enseignants (même quand il y en a, l'électricité et certaines commodités y font défaut, obligeant ainsi les enseignants à loger souvent très loin de leur lieu de travail); quasi-absence de prise en charge du transport; établissements ne répondant pas aux normes éducatives (insuffisance d'enseignants, absence d'encadrement, etc.). En réalité, cette situation s'explique par un relatif désintérêt des populations pour la scolarisation dû à la pauvreté et à des facteurs socioculturels (Koffi, 2014; Kouamé, 2014). 
Les résultats présentés ci-dessus corroborent ceux de recherches antérieures (Éla, 1998; Kouadio, 2001; Kouassi, Toh et Gono,2006) qui indiquent que la période avant l'avènement des réformes internationales était caractérisée par des initiatives de développement du secteur de l'éducation et particulièrement par des actions en faveur de la promotion de la profession enseignante en Côte d'Ivoire. Cette période, nostalgique et symbolique pour les organisations des enseignants dans la mesure où elle continue de marquer l'idéal de la lutte pour l'amélioration des conditions de travail et de vie des enseignants, constitue les repères de gloire de la profession enseignante en Côte d'Ivoire (Piot, 2010).

Cependant, la récession économique du début des années 1980 va mettre un terme à l'évolution des salaires des enseignants. Pis, les réformes politiques et économiques initiées par les institutions financières internationales pour améliorer la situation du pays vont amener à un " raccrochage » des salaires se traduisant par une discrimination salariale criarde dans le secteur de l'éducation, au point où on parlera en Côte d'Ivoire de « salaires à double vitesse ». Cette situation a négativement et fortement marqué la conscience collective ivoirienne en général et les enseignants particulièrement. Elle a contribué à accélérer la dégradation des conditions de vie d'une proportion importante des enseignants et a eu des incidences sur la pauvreté dans le secteur de l'éducation (Dembélé, 2005).

\section{La profession enseignante dans le contexte des réformes internationales en Côte d'Ivoire : de la période de disgrâce à la précarisation et à la déprofessionnalisation de la profession enseignante}

En général, les politiques transnationales, caractérisées par des programmes d'ajustement structurel, ont été synonymes de stagnation ou de baisse de la croissance économique, à tel point que les années 1980 ont été qualifiées de « décennies perdues " pour l'Afrique au sud du Sahara (Dowo, 1993; Ehui, 2008). C'est dire que les résultats de ces politiques n'ont pas été encourageants (Gassama, 2008; Wautelet, 1987), car ils «bloquent la croissance et accélèrent la dégradation des conditions de vie en dépit de la rhétorique sur la dimension sociale de l'ajustement structurel »(Éla, 1998, p. 342).

En Côte d'Ivoire, l'austérité imposée par la Banque mondiale et le Fonds monétaire international s'est traduite par la chute de l'enveloppe budgétaire consacrée au secteur de l'éducation, un secteur que ces deux institutions considéraient comme étant budgétivore et non prioritaire dans la politique de relance économique (Bessis, 1988; Éla, 1994). Selon les propos de M. T. L., ex-coordinateur national des activités extrascolaires du ministère de l'Éducation nationale, de 1982 à 1989, le budget du secteur de l'éducation a chuté régulièrement en moyenne de $35 \%$. Cette situation va s'empirer en 1990 avec une chute additionnelle de plus de 10 milliards de francs CFA, soit environ 20 millions de dollars US (Kouassi et al., 2006).

Quel impact cette chute du budget consacré à l'Éducation, synonyme du désintérêt pour le secteur, a-t-elle eu sur le système éducatif en général et particulièrement sur la profession enseignante? Cela nous conduit, dans une perspective compréhensive et analytique, à nous interroger sur les logiques des acteurs cibles pour saisir les déclinaisons de la profession enseignante dans un contexte de réformes internationales imposées par des institutions extérieures. 
En d'autres termes, selon les enquêtés, les réformes politiques et économiques initiées par la Banque mondiale et le Fonds monétaire international en Côte d'Ivoire n'ont pas eu d'effets positifs réels sur la profession enseignante. En effet, les données présentées au tableau 7 indiquent bien que les réformes initiées par les institutions financières internationales ont eu un impact très négatif sur les infrastructures éducatives du pays, selon les enquêtés. En effet, pour 86,51 \% d'eux, les infrastructures scolaires nont guère connu d'entretien depuis ces réformes, au point où plusieurs établissements scolaires sont restés presque inadéquats. Selon M.K.Y.S., ex-chef de cabinet au ministère de l'Éducation nationale, "les établissements éducatifs sont restés délabrés, vétustes depuis l'avènement de ces réformes en Côte d'Ivoire. Aujourd'bui, l'État cède aux Conseils généraux de départements et de districts l'entretien et la construction d'établissements pour corriger ces insuffisances engendrées par ces réformes».

Tableau 6

Appréciations du niveau de couverture des besoins en infrastructures par les instituteurs enquêtés.

\begin{tabular}{lcc}
\hline Appréciation & Valeur absolue & Fréquence \\
Mauvais & 109 & 86,51 \\
Bon & 17 & 13,49 \\
Total & $\mathbf{1 2 6}$ & $\mathbf{1 0 0 , 0}$ \\
\hline
\end{tabular}

L'étude des implications des politiques transnationales dans le secteur de l'éducation a conduit à l'analyse de l'inadéquation entre le nombre et les besoins en enseignants conduisant au déficit d'attractivité de la profession enseignante et à la fuite des cerveaux. Les résultats indiquent une grande influence des politiques internationales sur l'effectif des enseignants. En effet, selon le tableau 7,96,83 \% des enquêtés sont d'avis que les réformes des institutions financières internationales ont sonné le glas du recrutement d'enseignants où, dans plusieurs régions et localités du pays, des insuffisances d'enseignants sont notées dans des établissements primaires. Kouassi et al. (2006) et Toh et Gauthier (2011) précisent même que plusieurs établissements en Côte d'Ivoire ne disposent que de deux enseignants pour six classes, ce qui requiert qu'ils consentent de grands efforts pour prendre en charge les classes sans maîtres et complique davantage leurs tâches et les rend inefficaces, alors que le traitement salarial reste le même. Bien évidemment, cette situation ne prévoit aucun avantage complémentaire pour l'enseignant ivoirien au nom des contraintes budgétaires imposées par les réformes, mais également joue sur la qualité de l'éducation. Selon M.Z. U., responsable de section syndicale à l'école primaire publique Samaké d'Abobo à Abidjan :

La démotivation ou le manque d'attractivité de la fonction enseignante est fortement lié à la situation salariale des enseignants en Côte d'Ivoire. Depuis les années 90, les conditions de vie étant mauvaises, les maîtres ne se mettent réellement plus à la tâche dans la mesure de leurs capacités. Cela se ressent dans l'enseignement et surtout sur les résultats de fin d'année et le niveau des élèves. Dans notre jargon, on dit "petit argent, petit travail». Cela justifie également l'insuffisance d'enseignants dans la mesure [où] il y a la fuite des cerveaux.

En effet, selon le Document stratégique de réduction de la pauvreté (Ministère d'État chargé de la Planification et du Développement, 2009), les politiques internationales ont conduit à la baisse des dépenses publiques courantes, passant ainsi de 36,6 \% en 1990-1991 à 32,5\% en 1999-2000, soit 6,9\% du PIB en 1990 contre 3,9\% en 2000; et depuis 2002, la part du budget alloué à ces dépenses est restée constante et se situe autour de $22 \%$. 
En outre, le déficit d'attractivité de la profession enseignante marqué par le détournement de plusieurs jeunes diplômés ou enseignants du métier d'enseignant à tous les niveaux de l'enseignement. S'il est fait mention de la fuite des cerveaux du fait du désintérêt et du manque de motivation dans l'enseignement primaire, il est de même dans l'enseignement supérieur, où en 1998-1999, sur 108 postes budgétaires créés, seuls sept postes ont été pourvus (Ministère de la Fonction publique et de l'Emploi, 2000). Sur ces sept enseignants recrutés, on ne retrouve aujourd'hui qu'un seul et les six autres se sont expatriés au Canada et en Europe (SYNARES, 2010).

\section{Tableau 7}

Appréciation du rapport enseignants / besoins en enseignants.

\begin{tabular}{lcc}
\hline Appréciation & Valeur absolue & Fréquence \\
Mauvais & 122 & 96,83 \\
Bon & 4 & 3,17 \\
Total & $\mathbf{1 2 6}$ & $\mathbf{1 0 0 , 0}$ \\
\hline
\end{tabular}

\section{Les conditions de vie des enseignants dans le contexte des programmes d'ajustement structurel}

Les conditions de vie des enseignants sont abordées sous l'angle de la possibilité d'épargne qu'offre le traitement salarial des enseignants dans l'environnement socioéconomique marqué par les programmes d'ajustement structurel.

\section{Tableau 8}

Possibilité ou non d'épargne des instituteurs en période de politiques d'ajustement structurel.

\begin{tabular}{lcc}
\hline Opinion & Valeur absolue & Fréquence \\
Pas d'épargne & 96 & 76,19 \\
Épargne & 30 & 23,81 \\
Total & $\mathbf{1 2 6}$ & $\mathbf{1 0 0 , 0}$ \\
\hline
\end{tabular}

Les résultats présentés dans le tableau 8 traduisent l'impossibilité pour la majeure partie des enseignants enquêtés d'épargner dans un contexte d'austérité économique et financière. Comparativement aux données du tableau 5, cette situation traduit bien les difficiles conditions de vie socioéconomiques des enseignants dans la période des programmes d'ajustement structurel, qui a eu comme point culminant la réduction des salaires des nouveaux enseignants en 1991, créant ainsi une différenciation salariale et sociale des enseignants, mais également un discrédit de la profession enseignante en Côte d'Ivoire. Aujourd'hui, même si la situation a quelque peu évolué avec le "décrochage ", elle n'a pas fondamentalement changé la donne, du fait de l'inflation constante du coût de la vie alors que les salaires restent bloqués. Cela grève considérablement le pouvoir d'achat du personnel enseignant et accroît ainsi le risque de vulnérabilité de ceux-ci aux conjonctures économiques et sociales. Par exemple, devant un accroissement brutal du coût de la vie (augmentation des loyers, du prix des denrées alimentaires, du coût du transport, des soins médicaux, des fournitures scolaires, etc.), les enseignants 
sont dans l'incapacité de subvenir aux besoins primaires de leurs familles (Ehui, 2008). Les entrevues ont indiqué que, pour la plupart des enseignants, les réformes internationales ont été la source de toutes les difficultés qui ont contribué à accroître leur degré de vulnérabilité en les exposant davantage aux risques d'insécurité de leurs conditions de vie et de travail. D'ailleurs, des études (Bahlou Kophy, 2006; Kom, 2007) indiquent même que le milieu enseignant est très vulnérable à la pandémie du VIH/ SIDA. En effet, jusqu'en 2000 en Côte d'Ivoire, au moins deux enseignants décédaient par semaine de cette maladie (Kouassi et al., 2006). C'est en fait l'état de pauvreté des enseignants qui participe à cette vulnérabilité au VIH/SIDA dans le milieu enseignant et favorise par ailleurs la violence d'élèves à l'égard des enseignants (Vanga, Kouacou et Sika, 2006). Pour $\mathrm{M}^{\mathrm{me}} \mathrm{O}$. Y. C., directrice de l'école primaire publique de Cocody-Angré 1 :

la pauvreté des enseignants et la démotivation professionnelle a détruit la bonne perception
de la profession enseignante dans notre établissement et généralement en Côte d'Ivoire.
Les enseignants sont régulièrement endettés auprès des usuriers véreux. [...] En plus, les
conditions dans lesquelles ils vivent les rendent vulnérables à certaines maladies telles
que le VIH/SIDA, la tuberculose, les infections pulmonaires, les diarrhées chroniques, etc.
Plusieurs cas de maladies d'enseignants ont été révélés dans notre établissement et dans ceux
de plusieurs inspections à Abidjan, ainsi qu'à l'intérieur du pays où je travaillais. À cette
vulnérabilité s'ajoutent les violences sur les enseignants dans les établissements perpétrés
par les responsables de syndicats d'élèves. C'est bien sûr cette pauvreté des enseignants qui a
conduit à fragiliser l'image de la profession qui justifie ces violences-là...

En somme, on note que la situation d'épargne des enseignants laisse entrevoir des conditions de vie ou de construction socioéconomique à celles de leurre ou de la déconstruction socioéconomique de la profession enseignante, exprimant ainsi une dégradation ou une précarisation de la profession. Face à cette dégradation et à cette précarisation de la profession enseignante, quelles sont les mesures initiées par l'État pour corriger les imperfections nuisibles à la qualité de l'éducation en Côte d'Ivoire?

Les actions de l'État pour réduire l'impact des réformes transnationales sur la profession enseignante Les impacts négatifs des politiques liées aux mesures d'ajustement structurel ont fait émerger la problématique de la pauvreté des populations dans les pays ayant appliqué ces mesures (Éla, 1998; Gassama, 2008). Face à cette situation, les mécanismes de la résorption de la pauvreté sont devenus progressivement une priorité politique. En Côte d'Ivoire, même si les institutions financières internationales redoutent la croissance de la masse salariale, la problématique du devenir du social soulevé par la société civile a amené l'État à prendre des initiatives dans certains domaines sociaux, notamment l'éducation en vue d'atténuer l'effet de la précarisation continue de la profession enseignante. Il a donc mis à profit les marges de manœuvre que lui offrait l'amélioration du taux de croissance économique nationale (passé de -2,5\% en 1991 à 6,9 \% en 1996-1997) pour entreprendre des actions (Kouassi et al., 2006). Ces actions peuvent se résumer à (i) la concertation nationale sur l'école ivoirienne, (ii) la mise en place d'un plan national de développement de l'éducation et de la formation et (iii) plusieurs autres mesures spécifiques (Toh et Gauthier, 2011).

Face aux problèmes du système éducatif, le gouvernement a organisé une concertation nationale sur l'école ivoirienne regroupant les différents partenaires. Ces assises, dont les conclusions ont permis d'élaborer le projet de loi sur l'enseignement adopté par le Parlement en août 1995 et promulgué le 
7 septembre 1995 par le Président de la République, ont mis l'accent entre autres sur la valorisation socioéconomique de la profession enseignante par la revalorisation des salaires et du statut des enseignants. Pour ce faire, le pays s'est doté en 1997 d'un Plan national de développement de l'éducation et de la formation (PNDEF) pour la période 1998-2010. D'ailleurs, le gouvernement ivoirien recevra l'appui de la Banque mondiale et de plusieurs partenaires au développement pour la mise en œuvre de ces mesures en réponse aux incohérences et aux difficultés nées des réformes internationales dans le secteur de l'Éducation. Ces différentes mesures, qui traduisent la volonté du pays de réformer l'école en la sortant de la précarité, ont été accompagnées d'importantes décisions concernant les mesures d'accompagnement visant à améliorer le système éducatif ivoirien (Toh et Gauthier, 2011).

Si ces décisions ont été prises pour améliorer le système scolaire et la qualité de l'éducation, le constat sur le terrain montre bien que des mesures ne sont pas réellement exécutées et sont contournées par les responsables d'établissement. Cette réalité s'inscrit bien dans une stratégie de survie d'une profession enseignante en déconstruction. En effet, la précarité des conditions de vie des enseignants et la dégradation de l'environnement scolaire contraignent les responsables d'établissement et les enseignants (avec la complicité des comités de gestion des écoles) à imposer des cautions financières se situant entre 250 et 500 FCFA (entre 0,50 et 1 \$ US) dans les villages et entre 1250 et 5500 FCFA en moyenne (entre 2,5 et 11 \$US) dans les villes en ce qui concerne l'octroi des manuels scolaires aux élèves du primaire public. Aussi, au niveau des inscriptions, des frais non officiels allant de 100 à 1000 FCFA (de 0,20 à 2 \$ US) en zone rurale et de 2000 à 7500 FCFA (de 4 à 15 \$ US) en milieu urbain ont été notés. En plus, tous les enseignants organisent des séances dites de « renforcement » quasi obligatoires payantes aux horaires ou jours non ouvrés aux élèves du primaire. Le coût de la participation à ces séances varie entre 1000 et 5000 FCFA (de 2 à 10 \$ US) par mois. Ces pratiques sociales, qui viennent davantage révéler la situation de précarité des enseignants et de leur profession, rendent compte pour ainsi dire de stratégies de survie et de résilience, mais également traduisent l'inefficacité des actions et mesures initiées par l'État ivoirien pour arriver à bout de plusieurs années de vulnérabilité de la profession enseignante. Depuis 2012, des recrutements directs d'étudiants ou d'élèves bacheliers se font par l'État ivoirien pour combler les déficits de maîtres dans les classes sans pour autant respecter les délais de formation des maîtres. Ces promus sont ensuite formés en quelques mois, au lieu de deux années, puis déployés pour tenir des classes. Ces enseignants, assimilés aux " enseignants contractuels » au Mali, au Burkina Faso, au Sénégal, etc. (Dembélé, 2005) « n’ont pas de numéro matricule » et sont rémunérés trois, voire quatre fois moins que les maîtres de la "fonction publique ». Cette mesure, qui sort des principes ordinaires d'exercice de la profession enseignante, contribue à accentuer le mépris dans l'imaginaire social au point où on s'y engage quand on ne trouve pas un emploi plus valorisé et mieux rémunéré.

\section{Conclusion}

$\mathrm{Au}$ terme de cette contribution, retenons que les politiques internationales implantées pour sortir les pays d'Afrique subsaharienne de la crise économique et sociale qu'ils vivaient les ont plutôt placés dans une situation de précarité accentuée. Près d'une vingtaine d'années plus tard, en dépit de quelques initiatives non moins importantes dans l'amélioration des conditions de vie et de travail du personnel enseignant, les observations indiquent que les effets de ces politiques semblent se traduire par des 
contraintes qui surplombent encore la profession enseignante et le système éducatif ivoirien dans son entièreté.

La présente contribution indique que la précarité de la profession enseignante s'est accentuée, mais surtout que cette profession a été déstabilisée, car ayant perdu ses repères. À l'analyse des résultats, dans le domaine de l'éducation, tout laisse à penser et à croire que les institutions financières internationales visaient autre chose que l'amélioration du système éducatif. En tout état de cause, les conséquences de cette dégradation et de cette précarisation de la profession enseignante sont énormes et touchent particulièrement le système éducatif ivoirien et le développement du pays à long terme.

C'est pourquoi il est important de lancer d'autres mesures pour répondre efficacement et durablement aux impacts sociaux, économiques et culturels des réformes internationales, et non proposer des réponses qui continuent de plonger la profession enseignante dans une précarité sans nom au moment même où le pays vient d'atteindre le point d'achèvement de l'initiative $\mathrm{PPTE}^{3}$; ce qui lui permet d'envisager la sortie de la dette. Si l'on veut voir réellement l'amélioration des conditions de vie et de travail des enseignants et entreprendre des actions en vue de la qualité de l'éducation et d'une profession enseignante stimulante, il faut «aller au-delà des schémas économicistes » (Éla, 1998, p. 343) pour promouvoir une réforme éducative adaptée (Bissonnette, Richard et Gauthier, 2005) et la bonne gouvernance. Toutes ces mesures ne peuvent en effet être efficaces que si elles se situent dans un cadre stratégique de développement durable qui permet de revaloriser les salaires des enseignants et de lutter contre l'impunité à l'école.

\section{Notes}

1 PAS : programmes d'ajustement structurel.

2 Comme indiqué dans la méthodologie, ces individus étaient des enseignants bien avant les réformes. C'est à juste titre qu'ils ont donné leurs points de vue sur la question.

3 Pays pauvre très endetté.

\section{Références}

Bahlou Kophy, A. (2006). Le VIH/SIDA et le corps des enseignants. Quels impacts sur le système éducatif ivoirien? Rapport d'enquête épidémiologique dans les régions de Daloa et Abengourou en République de Côte d'Ivoire. Repéré à http://download.ei-ie.org/docs/IRISDocuments/EI\%20Campaigns/EFAIDS\%20Programme/2007-00111-01-F.doc

Bamba, N., Contamin, B., Diomandé, K. et Koulibaly, M. (1992). Crise économique et Programmes d'ajustement structurel en Côte d'Ivoire. Dans Crises et Ajustements en Côte d'Ivoire. Les dimensions sociales et culturelles (p. 10-23). Repéré à http://horizon.documentation.ird.fr/exl-doc/pleins textes/pleins textes 7/carton01/010012158.pdf

Bissonnette, S., Richard, M. et Gauthier, C. (2005). Échec scolaire et réforme éducative. Quand les solutions proposées deviennent la source du problème. Québec, QC : Presses de l'Université Laval.

Carr-Saunders, A. M. et Wilson, P. A. (1933). The Professions. Oxford : Clarendon Press.

Confédération générale du travail. (2006). Rapport du séminaire sur la dette publique : dette et développement en Côte d'Ivoire. Abidjan.

Corcuff, P. (2007). Les nouvelles sociologies (2 éd.). Paris : Armand Colin. 
Dembélé, M. (dir.) (2005). Étude sur la formation et la gestion des enseignants du primaire en Afrique de l'Ouest (Burkina Faso, Mali, Niger et Sénégal). Rapport de synthèse comparative. Washington, DC : Banque mondiale.

Dowo, R., Y. (2004). Impact des Programmes d'ajustement structurel en Côte d'Ivoire. Paris : L'Harmattan.

Dubar, C. (2002). La socialisation. Construction des identités sociales et professionnelles (3e éd.). Paris : Armand Colin.

Durufé, G. (1988). L'ajustement structurel en Afrique (Sénégal, Côte d'Ivoire, Madagascar). Paris : Karthala.

Éla, J.-M. (1998). Innovations sociales et renaissance de l'Afrique noire. Les défis du monde d'en bas. Montréal, QC : L'Harmattan.

Gassama, I. (2008). Les politiques d'ajustement structurelles et leurs conséquences sur les crises sociales en Afrique. Repéré à http://ibrahimagassama.centerblog.net/4517626-Les-politiques-d-ajustement-structurelles-et-leurs-consequencessur-les-crises-sociales-en-Afrique

Godelier, M. (1984). L'idéel et le matériel. Paris : Fayard.

Koffi, A. P. (2014). Climat scolaire et rendement dans les établissements d'enseignement secondaire de Côte d'Ivoire (Thèse de doctorat inédite). Université Félix Houphouët-Boigny de Cocody.

Kom, D. (2007). Valorisation des enseignants au Cameroun. Rapport d'étude OCEF/CEFAN. Yaoundé.

Kouadio, A. K. (2001). Dette de la Côte d'Ivoire et défi dans la lutte contre la pauvreté. Abidjan : CIRES et Université de Cocody.

Kouamé, A. S. (2014). Politique internationale et résilience sociale dans les établissements secondaires en Côte d'Ivoire (Thèse de doctorat inédite). Université Félix Houphouët-Boigny de Cocody.

Kouassi, N. F., Toh, A. et Gono, K., C. (2006). Les PAS et la lutte contre la pauvreté en Côte d'Ivoire : cas de l'enseignement primaire public. Rapport d'étude. CPAE-SNEPPCI.

Kpoumié Monjap, A. D. (2005). Mondialisation économique et condition enseignante dans les pays en développement (PED) : le cas du Cameroun et de la Guinée Conakry (Mémoire de maîtrise, Université de Montréal). Repéré à https://papyrus. bib.umontreal.ca/xmlui/handle/1866/17841

Ministère de l'Éducation nationale. (2005). Rapport sur le secteur Éducation-Formation. Côte d'Ivoire.

Ministère d'État chargé de la Planification et du Développement. (2009). Document stratégique de réduction de la pauvreté (DSRP) - Côte d'Ivoire.

Piot, C. (2010). Nostalgia for the Future: West after the Cold War. Chicago : University of Chicago Press. http://dx.doi. org/10.7208/chicago/9780226669663.001.0001

Sahi, V. A. (2005). Une chronique de la révolution démocratique en Côte d'Ivoire (1989-1995). Paris : L'Harmattan.

Toh, A. et Gauthier, C. (2011). Impacts des programmes d'ajustement structurel sur le système éducatif ivoirien : Retour pour une analyse sociologique des conditions de vie et de travail des instituteurs de l'enseignement primaire public en Côte d'Ivoire. Journal a fricain de communication scientifique et technologique, 1657-1680.

Vanga, A. F., Kouacou, A. et Sika, L. (2006, mars). La violence à l'école en Côte d'Ivoire : quelle implication des syndicats d'étudiants et d'élèves. Communication présentée au colloque international Éducation, Violences, Conflits et Perspectives de paix en Afrique, Yaoundé.

Wautelet, J.-M. (1987). Endettement, ajustement structurel et politique de développement. Louvain-la-Neuve : CIDEP.

\section{Pour citer cet article}

Toh, A. (2017). Précarisation de la profession enseignante au primaire en Côte d'Ivoire. Formation et profession, 25(2), 20-34. http://dx.doi.org/10318162/fp.2017.335 